\title{
HIGHLIGHTS
}

\section{Long-term outcomes of living kidney donors are affected by race}

A new study has shown that the race of living kidney donors has an effect on their medical outcomes after organ donationblack and Hispanic donors are at an increased risk of chronic kidney disease (CKD), hypertension and diabetes mellitus. "Our study emphasizes that increased attention to health outcomes among demographically diverse kidney donors is needed," says lead author Krista Lentine from the Saint Louis University Center for Outcomes Research, USA.

Nearly $40 \%$ of kidney transplants in the USA are from living donors, yet the health outcomes after living kidney donation are not well described in diverse populations, as most studies include few non-white participants. Potential donors undergo medical evaluations to determine their suitability before organ donation, but monitoring of individuals after donation can be minimal. "After donation, the nationally mandated tracking of donors by the Organ Procurement and Transplant Network (OPTN) in the USA currently extends only 2 years," explains Lentine. "Incomplete reporting even to 2 years and donor loss to follow-up are common."
Lentine and colleagues integrated OPTN registry data with administrative records from a private health insurance provider to obtain information on the medical care and long-term outcome of individuals after donation, independent of their interactions with the transplant center and beyond the OPTN 2-year follow-up period (median 7.7 years after donation). The researchers identified postdonation diagnoses of CKD, hypertension and diabetes mellitus in 4,650 individuals who donated a kidney between 1987 and 2007, and had claimed postnephrectomy health insurance benefits between 2000 and 2007. They then compared disease prevalence patterns in these individuals with that of the general population, according to 2005-2006 US NHANES data.

$76.3 \%$ of kidney donors were white, $13.1 \%$ were black, $8.2 \%$ were Hispanic and $2.4 \%$ belonged to another race or ethnic group. Black and Hispanic donors had a higher risk of hypertension, diabetes mellitus requiring drug treatment and CKD than white donors, irrespective of socioeconomic status. The prevalence of hypertension in older and Hispanic

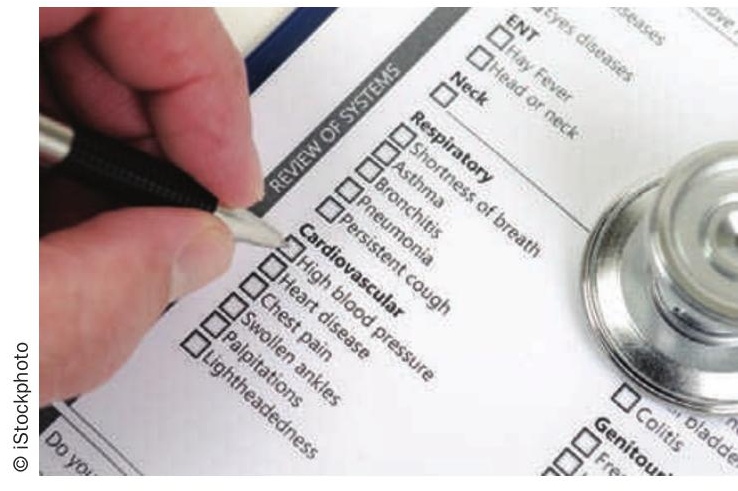

donors exceeded the NHANES estimate, but no difference was observed in the absolute prevalence of diabetes between all groups of donors and the general population.

"The lifetime risks of medical comorbidities, CKD and end-stage renal disease are increased for black and Hispanic individuals in general, and this seems to include non-white people who have donated kidneys," concludes Lentine. She advocates a longer and more reliable donor follow-up than is currently mandated in the USA, which she hopes will enable more donors from all sociodemographic groups to be monitored after donation in the long term.

Katrina Ray

Original article Lentine, K. L. et al. Racial variation in medical outcomes among living kidney donors. N. Engl. J. Med. 363, 724-732 (2010) 